\title{
Medicamentos potencialmente inapropriados para idosos e sua presença no SUS: Avaliação das Listas Padronizadas
}

\section{Pdentially Inaqpropriate Medictionfor Usein Edelly and itsPrevalenceintheSU: Evduation of theStandardzed lists of Dugs}

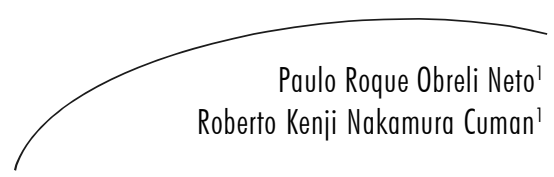

O objetivo deste trabalho foi verificar a existência de medicamentos potencialmente inapropriados para uso em idosos (PIM), e de alternativas farmacoterapêuticas mais seguras nas listas padronizadas municipais de medicamentos da atenção primária à saúde. Realizou-se estudo transversal, multicêntrico, exploratório, de natureza quantitativa nos 12 municípios que compõem a microrregião de Ourinhos, São Paulo, Brasil no período de maio a julho de 2009. Utilizamos como técnica a consulta a documentos (listas padronizadas municipais de medicamentos). O critério de Beers foi utilizado como ferramenta para classificação dos PIM. Foi encontrada alta prevalência de PIM, representando de $19,6 \%$ a $29,6 \%$ do número total de medicamentos padronizados nas listas padronizadas municipais Os PIM que atuam no sistema nervoso foram os mais prevalentes, seguidos pelos que atuam no sistema cardiovascular e no sistema músculo-esquelético, respectivamente. $\mathrm{O}$ número de PIM, constante das listas padronizadas municipais de medicamentos avaliadas, que apresentavam alguma alternativa farmacoterapêtica mais segura entre as especialidades farmacêuticas padronizadas, variou de $50,0 \%$ a $84,2 \%$ dos PIM, com um valor médio de $73,2 \%$. Esses resultados indicam a necessidade da adoção de estratégias como a implantação de protocolos clínicos para prescrição para idosos, mudanças no ensino médico e acompanhamento multiprofissional dos pacientes idosos, para evitar a prescrição de PIM para esta faixa da população.

\section{Abstract}

This study aims to verify the use potentially inappropriate medication in elderly people (PIM), and of safer pharmacotherapy choices in the municipal standard lists of drugs of the primary health care. A cross-sectional, multicenter, exploratory, quantitative study was conducted in the 12 municipalities that belong
Palavras-chave:

Farmacoterapia. Novas indicações de medicamentos. Idoso. Uso de Medicamentos. Saúde Pública. 
to the Ourinhos micro-region, Sao Paulo, Brazil, from May to July 2009. We used as technical consultation of documents (municipal standard lists of drugs). The Beers criteria was used to identify PIM. A high prevalence of PIM was found, with PIM representing $19.6 \%$ to $29.6 \%$ of the total number of drugs in the municipal standard lists. The PIM that acts in the nervous system were the most prevalent, followed by drugs that act on the cardiovascular system and skeletal muscle system, respectively. The number of PIM presenting safer pharmacotherapy alternatives in the standardized list studied ranged from $50.0 \%$ to $84.2 \%$ of the PIM, with a mean value of $73.2 \%$. These results indicate the need to adopt strategies as the implementation of clinical protocols for prescription to the elderly, changes in the medic education and multiprofessional follow-up of the elderly patients, to avoid the prescription of PIM to this range of the population.
Key words: Drug Therapy. Drug Repositioning. Health of the Elderly. Aged. Drug Utilization. Public Heath

\section{INTRODUÇÃO}

Nas últimas décadas, a população idosa no Brasil apresentou aumento considerável, com perspectivas de envelhecimento populacional acelerado para os próximos anos. ${ }^{1}$ Isto exige que o sistema público de saúde brasileiro (SUS) desenvolva e execute políticas, estratégias e ações que atendam às necessidades específicas deste crescente grupo populacional e garantam maior expectativa e qualidade de vida dos idosos.

O número de morbidades apresentadas pelos idosos aumenta, sobretudo em relação a doenças crônicas degenerativas, ${ }^{2}$ as quais geralmente requerem um número elevado de medicamentos para seu tratamento. ${ }^{3}$ Tal fato proporciona maior consumo de medicamentos pela população idosa, ${ }^{3-}$ ${ }^{5}$ expondo estes indivíduos a diversos problemas relacionados ao uso de medicamentos (PRM), ${ }^{6}$ ainda mais quando os medicamentos utilizados são potencialmente inapropriados para uso em idosos (PIM), apresentando risco elevado de ocorrência de reações adversas a medicamentos (RAM). ${ }^{7}$ Os PIM são definidos como medicamentos para os quais os riscos potenciais superam os benefícios esperados, e para os quais existe uma alternativa terapêutica mais segura disponível. ${ }^{8}$

A utilização de PIM pelos idosos consiste num dos maiores problemas de saúde pública atuais, por sua relação direta com o aumento da morbidade, mortalidade e gastos dos serviços de saúde. ${ }^{7,9}$ É imprescindível o desenvolvimento de estratégias que evitem a exposição da população idosa a PIM.

O uso de listas explícitas de PIM consiste numa estratégia viável para evitar a prescrição destes medicamentos, podendo ser destacados o Critério de Beers, ${ }^{10}$ o Critério Canadense ${ }^{11}$ e o Critério STOPP (Screening Tool of Older Persons' Potentially Inappropriate Prescriptions). ${ }^{12} \mathrm{O}$ Critério de Beers foi elaborado por um painel de especialistas norteamericanos (utilizando o método Delphi modificado) e tem sido extensamente utilizado na detecção de PIM, com implicações positivas nos resultados clínicos e econômicos da farmacoterapia em idosos. ${ }^{7}$ Sua versão atual consiste de duas listas de medicamentos (lista 1: independente do diagnóstico e condição clínica; e lista 2: considerando diagnóstico e condição clínica) que deveriam ser evitados para uso em idosos, classificados de acordo com grau de severidade das ADRs. ${ }^{10}$

No SUS, os medicamentos disponibilizados para a população são escolhidos mediante processos de seleção baseados no perfil de prevalência de doenças de uma localidade. Estas listas padronizadas são compostas de medicamentos com eficácia e segurança comprovada, que apresentem a melhor relação custo-benefício. ${ }^{13}$ Alguns dos medicamentos selecionados, embora efetivos e seguros para outros estratos etários da população, podem ser potencialmente inadequados para uso em idosos. Assim, é importante verificar a prevalência de PIM e a existência de alternativas mais seguras nas listas padronizadas de medicamentos dos municípios. 
O objetivo do presente trabalho foi verificar a existência de PIM e de alternativas farmacoterapêuticas mais seguras nas listas padronizadas municipais de medicamentos $\mathrm{da}$ atenção primária à saúde, com a finalidade de orientar melhor os prescritores na escolha terapêutica.

\section{METODOLOGIA}

Trata-se de um estudo transversal, multicêntrico, exploratório, de natureza quantitativa, realizado nos municípios pertencentes à microrregião de Ourinhos, Estado de São Paulo, Brasil, no período de maio a julho de 2009.

Essa microrregião é composta por 12 municípios, com população de 225.883 habitantes, sendo 28.034 habitantes pessoas com idade igual ou superior a 60 anos, segundo DATASUS (2007). Nesses municípios, o acesso a medicamentos na atenção básica à saúde tem como suporte os itens constantes das listas padronizadas municipais de medicamentos, que contêm os medicamentos selecionados pelas Secretarias Municipais de Saúde para atender toda a população, incluindo os indivíduos idosos.

Fizeram parte do estudo todos os municípios que compõem a microrregião de Ourinhos, Estado de São Paulo, Brasil. Utilizamos como técnica a consulta a documentos. Os documentos examinados foram as listas padronizadas municipais de medicamentos das Secretarias Municipais de Saúde. Tais listas são disponibilizadas para conhecimento dos profissionais de saúde e da população, nas Unidades Básicas de Saúde e sítios das prefeituras municipais; sendo um documento de domínio público. As listas padronizadas municipais de medicamentos foram confrontadas com o Critério de Beers, ${ }^{10}$ para verificar a presença de PIM e a existência de alternativas farmacoterapêuticas mais seguras, para serem utilizadas na população idosa. Esta análise foi realizada pelo número de PIM padronizado por município, dos PIM padronizados por município (agrupados de acordo com o sistema Anatomical - Therapentical Chemical Classification System 2009 (ATCC-2009) do Collaborating Center for Drugs Statistics Methodology da Organização Mundial da Saúde), ${ }^{14}$ do número de alternativas farmacoterapêuticas mais seguras existentes por município e da proporção de PIM em relação ao número total de medicamentos padronizados por município. Também foi verificada a presença de PIM nas listas de medicamentos constantes da Portaria $\mathrm{n}^{\circ} 3.237$, de 24 de dezembro de 2007 (Portaria n ${ }^{0}$ 3.237/ 2007) ${ }^{15}$ e do Programa Dose Certa do Governo do Estado de São Paulo (Programa Dose Certa). ${ }^{16}$

Os PIM existentes nas listas padronizadas municipais de medicamentos foram classificados em classes farmacológicas, de acordo com o ATCC-2009. Nessa classificação, os medicamentos são categorizados em diferentes grupos de acordo com o órgão ou sistema em que atuam e por suas propriedades químicas, terapêuticas e farmacológicas. ${ }^{14}$

A Portaria $n^{0} 3.237 / 2007$ aprova as normas de execução e de financiamento da assistência farmacêutica na atenção básica em saúde, dispondo o Elenco de Referência do Componente de Assistência Farmacêutica Básica, cuja aquisição será financiada pelos recursos das três esferas administrativas; delimitando em muitos casos os medicamentos que serão adquiridos pela assistência farmacêutica dos municípios. ${ }^{15}$

O Programa Dose Certa consiste na aplicação dos recursos estaduais padronizados pela Portaria $\mathrm{n}^{\mathrm{o}} 3.237 / 2007$, por meio da oferta de medicamentos (constantes do Elenco da Assistência de Referência do Componente de Assistência Farmacêutica Básica) produzidos pelo laboratório oficial: Fundação para o Remédio Popular (FURP); no sentido de fortalecer a produção pública de medicamentos. ${ }^{15,16}$

Os dados obtidos foram submetidos à análise estatística realizada por meio do Programa GraphPad Prism 5. Os dados foram apresentados como frequência e percentual.

As Secretarias Municipais de Saúde de todos os municípios, que integraram a amostra, foram 
informadas sobre os objetivos e procedimentos, e os responsáveis permitiram a inclusão dos dados de seu município na pesquisa. Cumpriram-se, na execução deste estudo, os princípios legais conforme versa a Resolução n ${ }^{\circ} 196 / 96$ do Conselho Nacional de Saúde, respeitando todos os aspectos éticos necessários para pesquisas desta natureza.

\section{RESULTADOS}

Foram verificadas 55 apresentações farmacêuticas classificadas como PIM pelo critério de Beers, ${ }^{10}$ parte das listas padronizadas municipais de medicamentos dos municípios analisados (tabela 1). Classificados de acordo com o sistema ATCC2009: 1 atua como antinfeccioso de uso sistêmico, 12 atuam no sistema cardiovascular, 12 atuam no sistema músculo-esquelético, 19 no sistema nervoso, três no sistema respiratório e oito no trato alimentar e metabolismo (tabela 1). Ocorreram desde casos de medicamentos padronizados em apenas um município ( $\mathrm{n}=3$ medicamentos), até medicamentos padronizados nos 12 municípios ( $\mathrm{n}=15$ medicamentos).

Tabela 1 - Medicamentos potencialmente inapropriados para uso em idosos nas listas padronizadas municipais, microrregião de Ourinhos, São Paulo, Brasil, 2009.

\begin{tabular}{|c|c|}
\hline Medicamento* & $\begin{array}{l}\text { Número de municípios em que o medicamento } \\
\text { é padronizado }\end{array}$ \\
\hline \multicolumn{2}{|l|}{ Anti-infecciosos de Uso Sistêmico } \\
\hline Nitrofurantoína comprimido $100 \mathrm{mg}$ & 1 \\
\hline \multicolumn{2}{|l|}{ Sistema Cardiovascular } \\
\hline Amiodarona comprimido $100 / 200 \mathrm{mg}$ & 10 \\
\hline Clonidina comprimido $0,150 \mathrm{mg}$ & 3 \\
\hline Digoxina comprimido $0,25 \mathrm{mg}$ & 12 \\
\hline Diltiazem comprimido $30 / 60 \mathrm{mg}$ & 8 \\
\hline Doxazosina comprimido $2 / 4 \mathrm{mg}$ & 6 \\
\hline Metildopa comprimido $250 / 500 \mathrm{mg}$ & 12 \\
\hline Propranolol comprimido $40 \mathrm{mg}$ & 12 \\
\hline Verapamil comprimido $80 \mathrm{mg}$ & 4 \\
\hline \multicolumn{2}{|l|}{ Sistema Músculo-esquelético } \\
\hline Ácido Acetilsalisílico comprimido 100/500 mg & 12 \\
\hline Cetoprofeno comprimido $100 \mathrm{mg}$ e injetável $50 \mathrm{mg} / \mathrm{mL}$ & 4 \\
\hline $\begin{array}{l}\text { Diclofenaco comprimido } 50 \mathrm{mg} \text {, injetável } 75 \mathrm{mg} / 3 \mathrm{~mL} \text { e } \\
\text { solução oral } 15 \mathrm{mg} / \mathrm{mL}\end{array}$ & 12 \\
\hline Ibuprofeno solução oral $100 \mathrm{mg} / \mathrm{mL}$ & 2 \\
\hline Nimesulida comprimido $100 \mathrm{mg}$ e solução oral $50 \mathrm{mg} / \mathrm{mL}$ & 7 \\
\hline Piroxicam comprimido $10 / 20 \mathrm{mg}$ & 3 \\
\hline \multicolumn{2}{|l|}{ Sistema Nervoso } \\
\hline Amitriptilina comprimido $25 \mathrm{mg}$ & 12 \\
\hline Clorpromazina comprimido $25 / 100$ mg e solução oral $4 \%$ & 12 \\
\hline
\end{tabular}


Tabela 1 - Medicamentos potencialmente inapropriados para uso em idosos nas listas padronizadas municipais, microrregião de Ourinhos, São Paulo, Brasil, 2009. (Continuação)

\begin{tabular}{|c|c|}
\hline Medicamento* & $\begin{array}{l}\text { Número de municípios em que o medicamento } \\
\text { é padronizado }\end{array}$ \\
\hline Diazepam comprimido $5 / 10 \mathrm{mg}$ & 12 \\
\hline Fenobarbital comprimido $100 \mathrm{mg}$, solução oral $40 \mathrm{mg} / \mathrm{mL}$ & 12 \\
\hline Fluoxetina comprimido $20 \mathrm{mg}$ & 12 \\
\hline $\begin{array}{l}\text { Haloperidol comprimido } 1 / 5 \mathrm{mg} \text {, solução oral } 2 \mathrm{mg} / \mathrm{mL} \text { e } \\
\text { haloperidol decanoato injetável }\end{array}$ & 12 \\
\hline Imipramina comprimido $25 \mathrm{mg}$ & 12 \\
\hline Metilfenidato comprimido $10 \mathrm{mg}$ & 1 \\
\hline Paroxetina comprimido $20 \mathrm{mg}$ & 1 \\
\hline Sertralina comprimido $50 \mathrm{mg}$ & 2 \\
\hline Tioridazina comprimido $50 / 100 \mathrm{mg}$ & 5 \\
\hline \multicolumn{2}{|l|}{ Sistema Respiratório } \\
\hline Dexclorfeniramina comprimido $2 \mathrm{mg}$ e xarope $2 \mathrm{mg} / 5 \mathrm{~mL}$ & 10 \\
\hline Prometazina comprimido $25 \mathrm{mg}$ & 12 \\
\hline \multicolumn{2}{|l|}{ Trato Alimentar e Metabolismo } \\
\hline $\begin{array}{l}\text { Butilbrometo de N-escopolamina comprimido } 10 \mathrm{mg} \mathrm{e} \\
\text { injetável } 20 \mathrm{mg} / \mathrm{mL}\end{array}$ & 9 \\
\hline Cimetidina comprimido $200 \mathrm{mg}$ e injetável $300 \mathrm{mg} / 2 \mathrm{~mL}$ & 12 \\
\hline Clorpropamida comprimido $250 \mathrm{mg}$ & 3 \\
\hline Metoclopramida comprimido $10 \mathrm{mg}$, solução oral $4 \mathrm{mg} / \mathrm{mL}$ & 12 \\
\hline Óleo mineral & 4 \\
\hline
\end{tabular}

* Os medicamentos estão dispostos de acordo com o sistema Anatomical - Therapeutical - Chemical Classification (ATCC) System 2009 do Collaborating Center for Drugs Statistics Methodology da Organização Mundial da Saúde.

A prevalência de PIM, classificada segundo Critério de Beers, ${ }^{10}$ nas listas padronizadas municipais de medicamentos dos municípios que compõem a amostra do estudo, variou de 19,6\% a 29,6\% dos medicamentos padronizados, com prevalência média de $23,1 \%$ dos medicamentos padronizados. O maior e o menor número de PIM observados nas listas padronizadas municipais de medicamentos foram dez e 42 especialidades farmacêuticas, respectivamente, com um valor médio de 31,6 PIM por município (tabela 2). 
Tabela 2 - Prevalência de medicamentos potencialmente inapropriados para uso em idosos nas listas padronizadas municipais, microrregião de Ourinhos, São Paulo, Brasil, 2009.

\begin{tabular}{|c|c|c|c|}
\hline Município & $\begin{array}{l}\text { Medicamentos padronizados } \\
\text { (n) }\end{array}$ & PIM padronizados (n) & $\begin{array}{c}\text { Prevalência de PIM } \\
(\%)\end{array}$ \\
\hline Município 1 & 123 & 28 & 22,7 \\
\hline Município 2 & 48 & 10 & 20,8 \\
\hline Município 3 & 125 & 37 & 29,6 \\
\hline Município 4 & 164 & 41 & 25,0 \\
\hline Município 5 & 143 & 28 & 19,6 \\
\hline Município 6 & 112 & 29 & 25,9 \\
\hline Município 7 & 206 & 42 & 20,4 \\
\hline Município 8 & 160 & 37 & 23,1 \\
\hline Município 9 & 148 & 34 & 23,0 \\
\hline Município 10 & 119 & 26 & 21,8 \\
\hline Município 11 & 152 & 38 & 25,0 \\
\hline Município 12 & 142 & 30 & 21,1 \\
\hline
\end{tabular}

O número de PIM, constante das listas padronizadas municipais de medicamentos avaliadas, que apresentavam alguma alternativa farmacoterapêutica maissegura entre as especialidades farmacêuticas padronizadas, variou de 50,0\% a $84,2 \%$ dos PIM, com um valor médio de $73,2 \%$ (tabela 3). O número de PIM constante das listas padronizadas municipais de medicamentos avaliados que não apresentavam alguma alternativa terapêutica mais segura entre as apresentações farmacêuticas padronizadas variou de $15,8 \%$ a 50,0\% dos PIM, com um valor médio de $26,8 \%$ (tabela 3 ).

Tabela 3 - Prevalência de alternativas farmacoterapêuticas mais seguras nas listas padronizadas municipais, microrregião de Ourinhos, São Paulo, Brasil, 2009.

\begin{tabular}{lccc}
\hline Município & $\begin{array}{c}\text { Número de PIM que } \\
\text { apresentam alternativas } \\
\mathrm{n}(\%)\end{array}$ & $\begin{array}{c}\text { Número de PIM que não } \\
\text { apresentam alternativas } \\
\mathrm{n}(\%)\end{array}$ & $\begin{array}{c}\text { Número total de PIM por } \\
\text { munić́pio }\end{array}$ \\
\hline Município 1 & $14(50,0)$ & $14(50,0)$ & 28 \\
Município 2 & $6(60,0)$ & $4(40,0)$ & 10 \\
Município 3 & $26(70,3)$ & $11(29,7)$ & 37 \\
Município 4 & $27(65,8)$ & $14(34,2)$ & 41 \\
Município 5 & $23(82,1)$ & $5(17,9)$ & 28 \\
Município 6 & $21(72,4)$ & $8(27,6)$ & 29 \\
Município 7 & $33(78,6)$ & $9(21,4)$ & 42 \\
Município 8 & $30(81,1)$ & $7(18,9)$ & 37 \\
Município 9 & $26(76,5)$ & $8(23,5)$ & 34 \\
Município 10 & $21(80,8)$ & $5(19,2)$ & 26 \\
Município 11 & $32(84,2)$ & $6(15,8)$ & 38 \\
Município 12 & $23(76,7)$ & $7(23,3)$ & 30 \\
\hline
\end{tabular}


Foi verificado que 29 apresentações farmacêuticas classificadas como PIM fazem parte de pelo menos um dos programas de financiamento e fornecimento de medicamentos da assistência farmacêutica dos municípios analisados (tabela 5). Dez apresentações farmacêuticas integram a Portaria $n^{\circ} 3237$, nove apresentações farmacêuticas integram o Programa Dose Certa e dez apresentações farmacêuticas integram ambos os programas (tabela 4).

Tabela 4 - Medicamentos potencialmente inapropriados para uso em idosos financiados e fornecidos, microrregião de Ourinhos, Estado de São Paulo, Brasil, 2009.

\begin{tabular}{|c|c|}
\hline Medicamento* & Programa de financiamento e fornecimento de medicamentos \\
\hline \multicolumn{2}{|l|}{ Sistema Cardiovascular } \\
\hline Digoxina comprimido $0,25 \mathrm{mg}$ & Portaria $n^{\circ} 3237$, de 24 de dezembro de 2007 e Programa D ose Certa \\
\hline Metildopa comprimido $250 \mathrm{mg}$ & Portaria $n^{\circ} 3237$, de 24 de dezembro de 2007 e Programa Dose Certa \\
\hline Propranolol comprimido 40mg & Portaria $n^{\circ} 3237$, de 24 de dezembro de 2007 e Programa Dose Certa \\
\hline Verapamil comprimido $80 \mathrm{mg}$ & Portaria no 3237 , de 24 de dezembro de 2007 \\
\hline \multicolumn{2}{|l|}{ Sistema Músculo Esquelético } \\
\hline $\begin{array}{l}\text { Ácido Acetilsalisílico comprimido } \\
\text { 100/500mg }\end{array}$ & Portaria $n^{\circ} 3237$, de 24 de dezembro de 2007 \\
\hline Diclofenaco comprimido $50 \mathrm{mg}$ & Programa Dose Certa \\
\hline Ibuprofeno solução oral $100 \mathrm{mg} / \mathrm{mL}$ & Portaria $\mathrm{n}^{\circ} 3237$, de 24 de dezembro de 2007 \\
\hline \multicolumn{2}{|l|}{ Sistema Nervoso } \\
\hline Amitriptilina comprimido $25 \mathrm{mg}$ & Portaria n ${ }^{\circ} 3237$, de 24 de dezembro de 2007 e Programa Dose Certa \\
\hline Clorpromazina comprimido $25 / 100 \mathrm{mg}$ & Portaria n 3237 , de 24 de dezembro de 2007 e Programa Dose Certa \\
\hline Clorpromazina solução oral 4\% & Portaria n ${ }^{\circ} 3237$, de 24 de dezembro de 2007 \\
\hline Diazepam comprimido $5 \mathrm{mg}$ & Portaria n ${ }^{\circ} 3237$, de 24 de dezembro de 2007 \\
\hline Diazepam comprimido $10 \mathrm{mg}$ & Programa Dose Certa \\
\hline Fenobarbital comprimido $100 \mathrm{mg}$ & Portaria $n^{\circ} 3237$, de 24 de dezembro de 2007 e Programa Dose Certa \\
\hline Fenobarbital solução oral $40 \mathrm{mg} / \mathrm{mL}$ & Portaria $\mathrm{n}^{\circ} 3237$, de 24 de dezembro de 2007 \\
\hline Fluoxetina comprimido $20 \mathrm{mg}$ & Programa Dose Certa \\
\hline Haloperidol comprimido $1 \mathrm{mg}$ & Portaria n ${ }^{\circ} 3237$, de 24 de dezembro de 2007 \\
\hline $\begin{array}{l}\text { Haloperidol comprimido } 5 \mathrm{mg} \text {, solução oral } \\
2 \mathrm{mg} / \mathrm{mL}\end{array}$ & Portaria $n^{\circ} 3237$, de 24 de dezembro de 2007 e Programa Dose Certa \\
\hline Haloperidol decanoato injetável & Programa Dose Certa \\
\hline Imipramina comprimido $25 \mathrm{mg}$ & Programa Dose Certa \\
\hline Sertralina comprimido $50 \mathrm{mg}$ & Programa Dose Certa \\
\hline Tioridazina comprimido $100 \mathrm{mg}$ & Programa Dose Certa \\
\hline \multicolumn{2}{|l|}{ Sistema Respiratório } \\
\hline $\begin{array}{l}\text { Dexclorfeniramina comprimido } 2 \mathrm{mg} \mathrm{e} \\
\text { xarope } 2 \mathrm{mg} / 5 \mathrm{~mL}\end{array}$ & Portaria $n^{\circ} 3237$, de 24 de dezembro de 2007 \\
\hline Prometazina comprimido $25 \mathrm{mg}$ & Programa Dose Certa \\
\hline \multicolumn{2}{|l|}{ Trato Alimentar e Metabolismo } \\
\hline Cimetidina comprimido $200 \mathrm{mg}$ & Programa Dose Certa \\
\hline Metoclopra mida comprimido $10 \mathrm{mg}$ & Portaria n ${ }^{\circ} 3237$, de 24 de dezembro de 2007 e Programa Dose Certa \\
\hline
\end{tabular}

* Os medicamentos estão dispostos de acordo com o sistema Anatomical - Therapeutical - Chemical Classification (ATCC) System 2009 do Collaborating Center for Drugs Statistics Methodology da Organização Mundial da Saúde. 


\section{DISCUSSÃO}

Estudos populacionais brasileiros verificaram prevalência média de $10 \%$ de PIM nas prescrições para indivíduos idosos. ${ }^{3,17}$ A maioria dos PIM prescritos para a população idosa são medicamentos que atuam no sistema cardiovascular $(28,4 \%$ a $47,4 \%$ de todos os medicamentos prescritos), no sistema nervoso $(12,3 \%$ a $21,5 \%$ de todos os medicamentos prescritos) e sistema músculo-esquelético $(6,2 \%$ a $15,0 \%$ de todos os medicamentos prescritos). $3,4,5,17$ Justamente estes medicamentos foram os encontrados com maior freqüência nas listas padronizadas dos municípios da microrregião estudada.

O processo de seleção de medicamentos é imprescindível para garantir acesso da população a medicamentos essenciais, por um valor viável para a administração pública. ${ }^{13}$ Os medicamentos componentes das listas padronizadas dos municípios analisados são em muitos casos a melhor opção farmacoterapêutica (relação custo, segurança, eficácia e comodidade de uso) para diversas faixas etárias da população. Entretanto, muitos dos medicamentos padronizados pelos municípios da microrregião de Ourinhos são considerados PIM. Isto gera a necessidade do desenvolvimento de estratégias que orientem os prescritores na escolha da terapêutica dos pacientes idosos.

Verificou-se a existência de alternativas terapêuticas mais seguras para os PIM na maioria dos casos das listas padronizadas. Isto possibilitará criar protocolos clínicos para escolha terapêutica para estes pacientes, o que poderá reduzir a prescrição de PIM para esta faixa da população, e permitir que outras faixas etárias continuem sendo beneficiadas por esses medicamentos. Esses protocolos clínicos devem ser amplamente divulgados e discutidos com os prescritores, para garantir que estes adotem esta estratégia. Processos de educação continuada dos prescritores e elaboração de formulários terapêuticos são imprescindíveis para uma boa implantação de protocolos clínicos. ${ }^{13}$
A inserção de disciplinas voltadas para a farmacoterapia racional no ensino médico consiste em outra estratégia viável e efetiva para adequação da escolha terapêutica para a população idosa. ${ }^{18}$ De Vries et al. ${ }^{18}$ verificaram resultado efetivo na implantação de curso de curta duração sobre farmacoterapia racional para estudantes de medicina.

Outra estratégia efetiva para redução das prescrições de PIM para idosos é o acompanhamento multiprofissional desses indivíduos. ${ }^{19}$ Obreli Neto \& Cuman ${ }^{19}$ verificaram redução significativa no consumo de PIM em pacientes acompanhados por um programa multiprofissional, envolvendo farmacêuticos, médicos e enfermeiros, em uma Unidade Básica de Saúde. Estas estratégias de acompanhamento multiprofissional permitem um atendimento integralizado e mais humanizado. ${ }^{20}$

Os programas de financiamento e fornecimento de medicamentos dos Governos Federal e estadual (Portaria no 3.237 e Programa Dose Certa, respectivamente), influenciam demasiadamente a elaboração das listas padronizadas municipais de medicamentos. Isto ocorre pelo fato de o financiamento da assistência farmacêutica na atenção básica ser executado, principalmente, por estes programas, sendo inviável para os municípios arcar com os custos e justificar a compra de medicamentos que não integrem as listas de medicamentos padronizados pela Portaria no 3.237 e o Programa Dose Certa.

Todos os casos de PIM que foram encontrados em todas as listas padronizadas municipais de medicamentos dos 12 municípios estudados (ácido acetilsalisílico, amitriptilina, cimetidina, clorpromazina, diazepam, diclofenaco, digoxina, fenobarbital, fluoxetina, haloperidol, imipramina, metildopa, metoclopramida, prometazina, propranolol) faziam parte de algum destes programas, com poucas alternativas mais seguras para substituir esses PIM.

Os resultados obtidos com a presente pesquisa demonstram a necessidade de revisão dos medicamentos que integram esses programas, para 
garantir a existência de alternativas mais seguras para a farmacoterapia da população idosa.

\section{CONCLUSÃO}

As listas padronizadas municipais de medicamentos das Secretarias Municipais de Saúde dos municípios que compõem a microrregião de Ourinhos apresentam número elevado de PIM, principalmente para o tratamento das morbidades que mais acometem a população idosa. Para a maioria dos PIM, existem alternativas farmacoterapêuticas mais seguras nas listas padronizadas.

\section{REFERÊNCIAS}

1. Instituto de pesquisa Econômica e aplicada ( IPEA). Comunicado da Previdência : demografia gênero. PNAD 2007;11(3). [acesso 15 ago 2009]. Disponível em: URL: http:// www.ipea.gov.br/sites/000/2/ comunicado_presidencia/ 08_09_30_Pnad_PrimeirasAnalises_N10MT.pdf.

2. Kilsztajn S, et al. Serviços de saúde, gastos e envelhecimento da população brasileira. Rev Bras Estud Popul 2003 jan./jun.; 20(1): 93-108.

3. Rozenfeld S, Fonseca MJM, de Assis Acurcio F. Drug utilization and polypharmacy among the elderly: a survey in Rio de Janeiro City. Rev Panam Salud Publica 2008 jan.; 23(1): 34-43.

4. Marin MJS, et al. Caracterização do uso de medicamentos entre idosos de uma unidade do programa saúde da família. Cad Saúde Pública 2008 jul.; 24(7): 1545-55.

5. Ribeiro $A Q$, et al. Inquérito sobre uso de medicamentos por idosos aposentados, MG. Rev Saúde Pública 2008 ago.; 42(4): 724-732.

6. Gurwitz JH. Polypharmacy: a new paradigm for quality drug therapy in the elderly? Arch Intern Med 2004 out.; 164: 1957-59.

7. Fick DM, et al. Health outcomes associated with potentially Inappropriate medication use in older adults. Res Nurs Health 2008 fev.; 31(1): 42-51.

8. Beers MH. Explicit criteria for determining potentially inappropriate medication use by the elderly: an update. Arch Intern Med 1997 jul.; 157(14): 1531-1536.
Isto possibilita o desenvolvimento de estratégias que promovam o uso racional de medicamentos, como a adoção de protocolos clínicos de prescrição para idosos, mudanças no ensino médico e acompanhamento multiprofissional dos pacientes idosos.

\section{AGRADECIMENTOS}

Agradecemos aos profissionais das Secretarias Municipais de Saúde dos municípios da microrregião Ourinhos, Estado de São Paulo, Brasil.

9. Spinewine A, et al. Appropriate prescribing in older people: how well can it be measured and optimized. Lancet 2007; 370: 173-184.

10. Fick D, et al. Updating the beers criteria for potentially inappropriate medication use in older adults. Arch Intern Med 2003 dez.; 163: 2716-2724.

11. McLeod PJ, et al. Defining inappropriate practices in prescribing for elderly people: a national consensus panel. CMAJ 1997; 156(3): 385-91.

12. Gallagher P, et al. STOPP (Screening Tool of Older Person's Prescriptions) and START (Screening Tool to Alert doctors to Right Treatment) :consensus validation. Int J Clin Pharmacol Ther 2008 fev.; 46(2):72-83.

13. Marin $\mathrm{N}$, et al. Seleção de medicamentos. In: Marin N, et al. Assistência farmacêutica para gerentes municipais. Rio de Janeiro: Organização Pan-Americana de Saúde/ Organização Mundial da Saúde; 2003. p 133-54.

14. World Health Organization. ATC/DDD Index 2009 [acesso 09 fev 2009]. Disponível em: URL: http://www.whocc.no/atcddd/

15. Ministério da Saúde (Brasil). Portaria $n^{\circ}$. 3.237, de 24 de dezembro de 2007. Aprova as normas de execução e financiamento da assistência farmacêutica na atenção básica em saúde. Diário Oficial da União 26 dez.

16. Secretaria de Estado da Saúde de São Paulo. Assistência Farmacêutica. : medicamentos do programa dose certa. Secretaria de Estado da Saúde de São Paulo;2009 [acesso 04 jul 2009]. Disponível em: URL: http:// www.farma.saude.sp.gov.br/. 
17. Coelho Filho JM, Marcopito LM, Castelo A. Perfil de utilização de medicamentos por idosos em uma área urbana do nordeste do Brasil. Rev Saúde Pública 2004 ago.; 38(4): 557-64.

18. De Vries TPGM, et al. Impact of a short course in pharmacotherapy for undergraduate medical students. Lancet 1995; 346: 1454-57.
19. Obreli Neto PR, Cuman RKN. Programa de atenção farmacêtica no uso racional de medicamentos em idosos usuários de unidade básica de saúde no estado de São Paulo. Am J Pharm 2010;29(3):333-39.

20. Lyra Júnior DP, et al. A farmacoterapia no idoso: revisão sobre a abordagem multiprofissional no controle da hipertensão arterial sistêmica. Rev Latino-am Enferm 2006; 14(3): 435-41.

Recebido: $17 / 12 / 2009$

Revisado: 04/11/2010

Aprovado: 06/12/2010 